-ELECTRONIC SUPPORTING INFORMATION (ESI)-

\title{
Temperature Effect on Activation Rate Constants in ATRP - New Mechanistic Insights into the Activation Process
}

\author{
Florian Seeliger and Krzysztof Matyjaszewski* \\ Center for Macromolecular Engineering, Department of Chemistry, Carnegie Mellon \\ University, 4400 Fifth Avenue, Pittsburgh, Pennsylvania 15213
}

km3b@andrew.cmu.edu

$\underline{\text { Table of contents }}$

$\begin{array}{ll}\text { General } & 2 \\ \mathrm{Cu}(\mathrm{I}) \mathrm{Br}(\mathrm{PMDETA})+\mathrm{TEMPO} \text { without initiator } & 2 \\ \mathrm{BrPN} & 3 \\ \mathrm{BrAN} & 3 \\ \mathrm{EtBriB} & \\ \mathrm{MBriB} & \\ \mathrm{ClPN} & \\ \mathrm{PEBr} & \\ \mathrm{ClAN} & \\ \mathrm{AllBr} & \\ \mathrm{MBrP} & \\ \text { Determination of } \Delta S^{\ddagger} & 10\end{array}$




\section{General}

The reactions were followed by using a Hellma immersion probe. The temperature was controlled in a jacketed beaker with a Haake K50 thermostat unit $\left(-25-+60{ }^{\circ} \mathrm{C}\right)$. Reactions at even lower temperatures were conducted in an acetone/dry ice bath. At $20{ }^{\circ} \mathrm{C}$ each initiator/catalyst system was studied at least three times at different initiator concentrations. Plots of the obtained pseudo-first-order rate constants yielded straight lines from which the activation rate constants were determined. Kinetic experiments at all other temperatures were only performed once.

\section{$\mathrm{Cu}(\mathrm{I}) \mathrm{Br}(\mathrm{PMDETA})+\mathrm{TEMPO}$ without initiator}

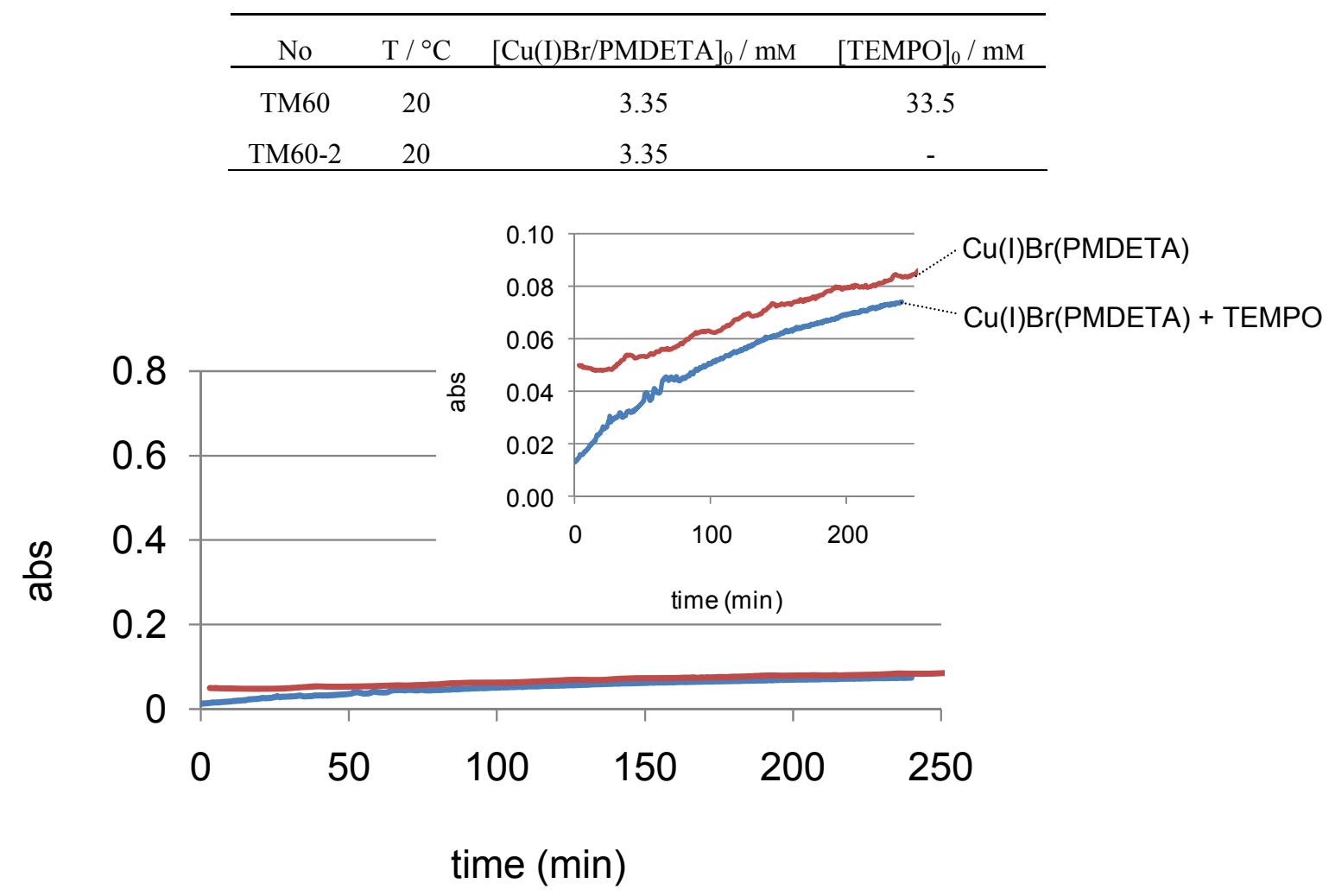

The experiments show the slow oxidation of the $\mathrm{Cu}(\mathrm{I}) \mathrm{Br}$ (PMDETA) complex by air. Addition of TEMPO does not influence this process. 


\section{BrPN}

$\mathrm{BrPN}$ is a very reactive initiator, which means that the reactions with $\mathrm{CuBr}$ (PMDETA) have to be performed under much diluted conditions and at low temperatures.

\begin{tabular}{ccccccc}
\hline No & $\mathrm{T} /{ }^{\circ} \mathrm{C}$ & {$[\mathrm{Cu}(\mathrm{I}) \mathrm{Br} / \mathrm{PMDETA}]_{0} / \mathrm{mM}$} & {$[\mathrm{BrPN}]_{0} / \mathrm{mM}$} & {$[\mathrm{TEMPO}]_{0} / \mathrm{mM}$} & $k_{\mathrm{obs}} / \mathrm{s}^{-1}$ & $k_{\mathrm{act}} / \mathrm{M}^{-1} \mathrm{~s}^{-1}$ \\
\hline FS145 & -41 & 1.68 & 17.3 & 17.2 & $1.82 \times 10^{-2}$ & 1.05 \\
FS146 & -36.5 & 1.68 & 17.0 & 17.2 & $2.35 \times 10^{-2}$ & 1.38 \\
FS154 & -39 & 1.24 & 12.7 & 13.4 & $1.45 \times 10^{-2}$ & 1.14 \\
FS155 & -32 & 1.24 & 12.7 & 13.4 & $2.29 \times 10^{-2}$ & 1.81 \\
FS156 & -28.5 & 1.25 & 12.9 & 13.5 & $2.65 \times 10^{-2}$ & 2.05 \\
FS157 & -23 & 1.25 & 12.9 & 13.5 & $3.61 \times 10^{-2}$ & 2.80 \\
FS158 & -15 & 1.25 & 12.9 & 13.5 & $5.99 \times 10^{-2}$ & 4.64 \\
\hline
\end{tabular}

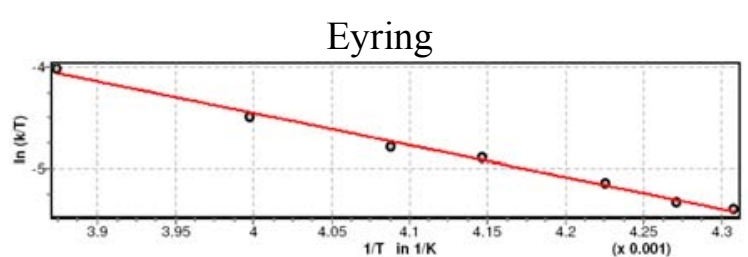

$$
\Delta H^{\ddagger}=(26.0 \pm 0.9) \mathrm{kJ} \mathrm{mol}^{-1}
$$$$
\Delta S^{\ddagger}=(-131 \pm 4) \mathrm{J} \mathrm{mol}^{-1} \mathrm{~K}^{-1}
$$

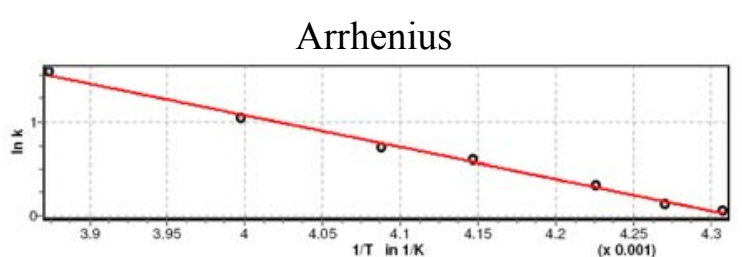

$$
E_{\mathrm{a}}=(28.1 \pm 0.9) \mathrm{kJ} \mathrm{mol}^{-1}
$$$$
\ln \mathrm{A}=14.6 \pm 0.4
$$

\section{BrAN}

\begin{tabular}{cccccc}
\hline No & $\mathrm{T} /{ }^{\circ} \mathrm{C}$ & {$[\mathrm{Cu}(\mathrm{I}) \mathrm{Br} / \mathrm{PMDETA}]_{0} / \mathrm{mM}$} & {$[\mathrm{BrAN}]_{0} / \mathrm{mM}$} & {$[\mathrm{TEMPO}]_{0} / \mathrm{mM}$} & $k_{\mathrm{obs}} / \mathrm{s}^{-1}$ \\
\hline FS237 & 20 & 0.98 & 10.1 & 12.2 & $3.69 \times 10^{-2}$ \\
FS238 & 20 & 0.97 & 13.0 & 12.0 & $4.66 \times 10^{-2}$ \\
FS239 & 20 & 0.95 & 16.2 & 11.8 & $5.87 \times 10^{-2}$ \\
FS247 & 20 & 1.06 & 19.0 & 13.2 & $7.18 \times 10^{-2}$ \\
\hline
\end{tabular}




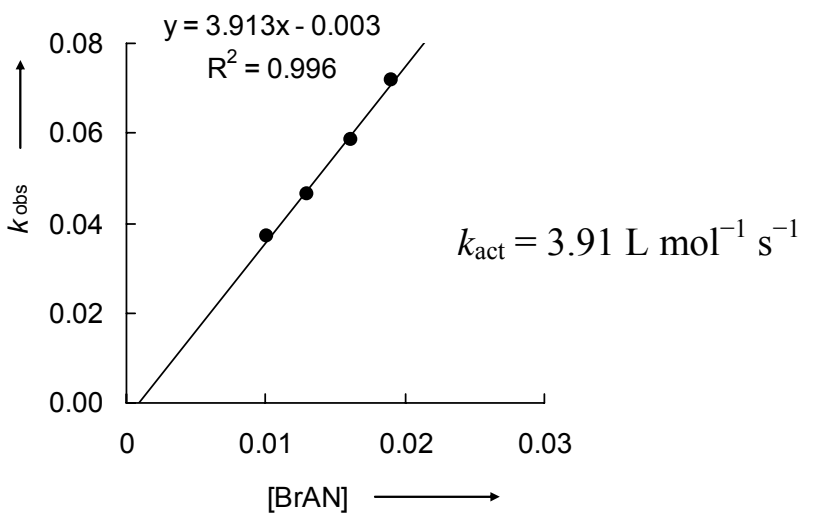

First-order kinetics:

\begin{tabular}{ccccccc}
\hline No & $\mathrm{T} /{ }^{\circ} \mathrm{C}$ & {$[\mathrm{Cu}(\mathrm{I}) \mathrm{Br}]_{0} / \mathrm{mM}$} & {$[\mathrm{PMDETA}]_{0} / \mathrm{mM}$} & {$[\mathrm{BrAN}]_{0} / \mathrm{mM}$} & {$[\mathrm{TEMPO}]_{0} / \mathrm{mM}$} & $k_{\mathrm{act}} / \mathrm{M}^{-1} \mathrm{~s}^{-1}$ \\
\hline $\mathrm{FS} 281$ & -40 & 3.43 & 3.43 & 36.1 & 34.3 & $1.40 \times 10^{-1}$ \\
\hline
\end{tabular}

Second-order kinetics:

\begin{tabular}{ccccccc}
\hline No & $\mathrm{T} /{ }^{\circ} \mathrm{C}$ & {$[\mathrm{Cu}(\mathrm{I}) \mathrm{Br}]_{0} / \mathrm{mM}$} & {$[\mathrm{PMDETA}]_{0} / \mathrm{mM}$} & {$[\mathrm{BrAN}]_{0} / \mathrm{mM}$} & {$[\mathrm{TEMPO}]_{0} / \mathrm{mM}$} & $k_{\mathrm{act}} / \mathrm{M}^{-1} \mathrm{~s}^{-1}$ \\
\hline FS70 & -20 & 4.21 & 4.21 & 4.21 & 42.6 & $4.93 \times 10^{-1}$ \\
FS72 & 0 & 3.90 & 3.90 & 3.90 & 38.9 & 1.56 \\
FS75 & 40 & 3.52 & 3.52 & 3.52 & 35.2 & 7.88 \\
\hline
\end{tabular}
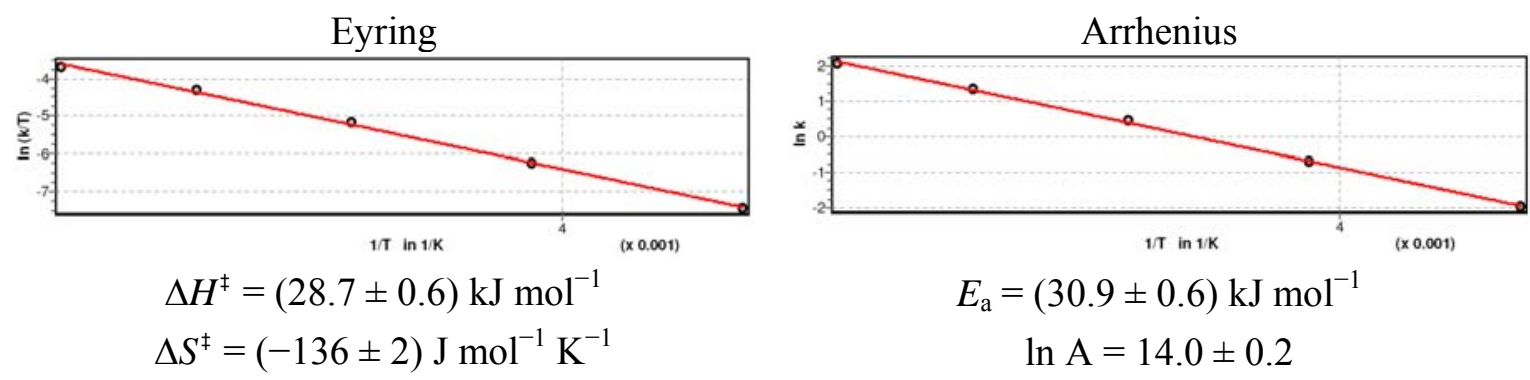

\section{EtBriB}

\begin{tabular}{cccccc}
\hline No & $\mathrm{T} /{ }^{\circ} \mathrm{C}$ & {$[\mathrm{Cu}(\mathrm{I}) \mathrm{Br} / \mathrm{PMDETA}]_{0} / \mathrm{mM}$} & {$[\mathrm{EtBriB}]_{0} / \mathrm{mM}$} & {$[\mathrm{TEMPO}]_{0} / \mathrm{mM}$} & $k_{\mathrm{obs}} / \mathrm{s}^{-1}$ \\
\hline FS240 & 20 & 1.25 & 12.8 & 15.5 & $2.10 \times 10^{-2}$ \\
FS241 & 20 & 1.23 & 18.9 & 15.2 & $3.11 \times 10^{-2}$ \\
FS242 & 20 & 1.12 & 23.8 & 13.9 & $4.11 \times 10^{-2}$ \\
FS243 & 20 & 1.10 & 29.4 & 13.7 & $4.97 \times 10^{-2}$ \\
\hline
\end{tabular}




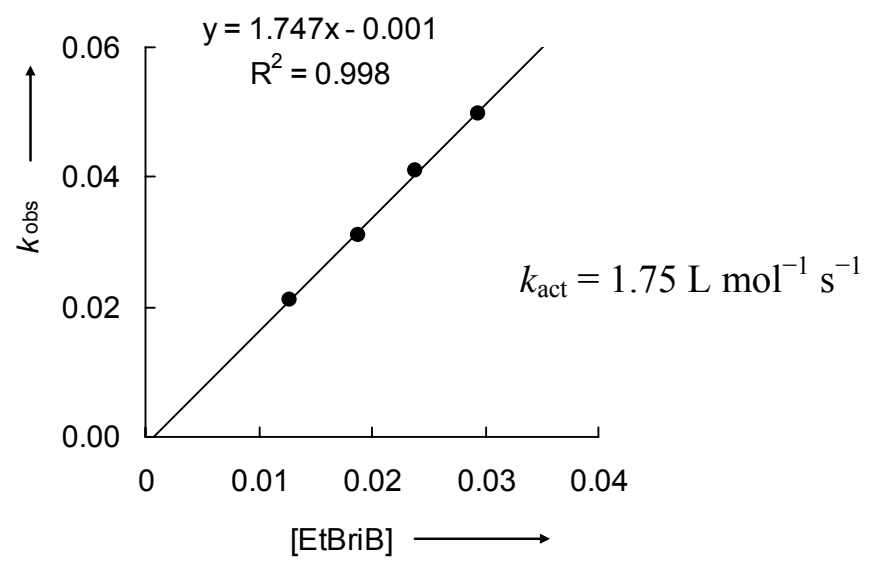

Second-order-kinetics:

\begin{tabular}{ccccccc}
\hline No & $\mathrm{T} /{ }^{\circ} \mathrm{C}$ & {$[\mathrm{Cu}(\mathrm{I}) \mathrm{Br}]_{0} / \mathrm{mM}$} & {$[\mathrm{PMDETA}]_{0} / \mathrm{mM}$} & {$[\mathrm{EtBriB}]_{0} / \mathrm{mM}$} & {$[\mathrm{TEMPO}]_{0} / \mathrm{mM}$} & $k_{\mathrm{act}} / \mathrm{M}^{-1} \mathrm{~s}^{-1}$ \\
\hline FS69 & -20 & 5.24 & 5.24 & 5.23 & 52.4 & $3.47 \times 10^{-1}$ \\
FS67 & 0 & 5.08 & 5.08 & 5.09 & 51.1 & $8.83 \times 10^{-1}$ \\
FS99 & 40 & 3.91 & 3.91 & 3.90 & 40.9 & 4.08 \\
\hline
\end{tabular}

First-order-kinetics:

\begin{tabular}{ccccccc}
\hline No & $\mathrm{T} /{ }^{\circ} \mathrm{C}$ & {$[\mathrm{Cu}(\mathrm{I}) \mathrm{Br} / \mathrm{PMDETA}]_{0} / \mathrm{mM}$} & {$[\mathrm{EtBriB}]_{0} / \mathrm{mM}$} & {$[\mathrm{TEMPO}]_{0} / \mathrm{mM}$} & $k_{\mathrm{obs}} / \mathrm{s}^{-1}$ & $k_{\mathrm{act}} / \mathrm{M}^{-1} \mathrm{~s}^{-1}$ \\
\hline \multirow{2}{*}{ FS68 } & 0 & 2.54 & 26.5 & 25.5 & $2.51 \times 10^{-2}$ & $9.48 \times 10^{-1}$ \\
FS282 & -40 & 3.35 & 33.4 & 33.5 & $3.35 \times 10^{-3}$ & $1.00 \times 10^{-1}$ \\
\hline
\end{tabular}

As can be seen from the two measurements at $0{ }^{\circ} \mathrm{C}$, both methods - second-order and pseudofirst-order approach - yield comparable values for $k_{\text {act. }}$.
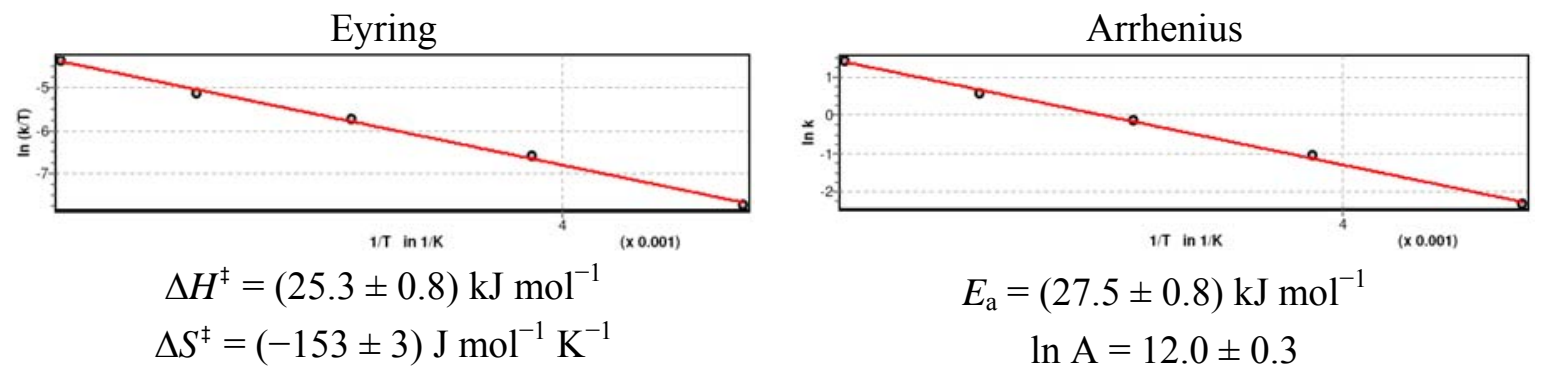

\section{MBriB}

\begin{tabular}{cccccc}
\hline No & $\mathrm{T} /{ }^{\circ} \mathrm{C}$ & {$[\mathrm{Cu}(\mathrm{I}) \mathrm{Br} / \mathrm{PMDETA}]_{0} / \mathrm{mM}$} & {$[\mathrm{MBriB}]_{0} / \mathrm{mM}$} & {$[\mathrm{TEMPO}]_{0} / \mathrm{mM}$} & $k_{\mathrm{obs}} / \mathrm{s}^{-1}$ \\
\hline FS244 & 20 & 1.21 & 12.4 & 15.0 & $2.91 \times 10^{-2}$ \\
$\mathrm{FS} 245$ & 20 & 1.18 & 17.0 & 14.7 & $3.63 \times 10^{-2}$ \\
FS126 & 20 & 1.85 & 19.0 & 19.5 & $4.26 \times 10^{-2}$ \\
FS246 & 20 & 1.06 & 21.8 & 13.2 & $5.16 \times 10^{-2}$ \\
\hline
\end{tabular}




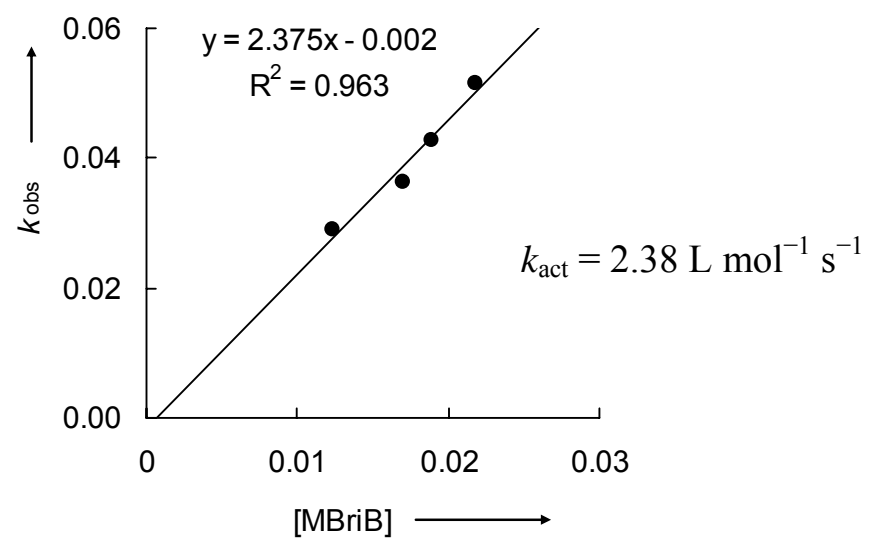

\begin{tabular}{ccccccc}
\hline No & $\mathrm{T} /{ }^{\circ} \mathrm{C}$ & {$[\mathrm{Cu}(\mathrm{I}) \mathrm{Br} / \mathrm{PMDETA}]_{0} / \mathrm{mM}$} & {$[\mathrm{MBriB}]_{0} / \mathrm{mM}$} & {$[\mathrm{TEMPO}]_{0} / \mathrm{mM}$} & $k_{\mathrm{obs}} / \mathrm{s}^{-1}$ & $k_{\mathrm{act}} / \mathrm{M}^{-1} \mathrm{~s}^{-1}$ \\
\hline $\mathrm{FS} 283$ & -40 & 3.20 & 33.4 & 32.0 & $4.04 \times 10^{-3}$ & $1.21 \times 10^{-1}$ \\
$\mathrm{FS} 124$ & -20 & 2.56 & 26.3 & 25.8 & $1.18 \times 10^{-2}$ & $4.49 \times 10^{-1}$ \\
$\mathrm{FS} 125$ & 0 & 2.64 & 27.1 & 27.8 & $3.04 \times 10^{-2}$ & 1.12 \\
$\mathrm{FS} 127$ & 40 & 1.71 & 17.2 & 17.7 & $8.06 \times 10^{-2}$ & 4.68 \\
\hline
\end{tabular}
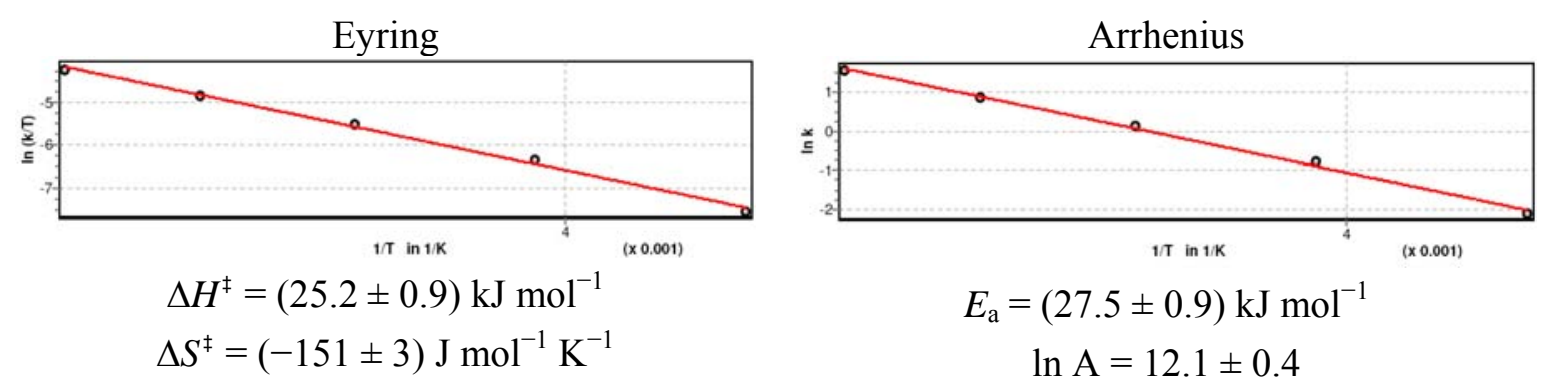

\section{CIPN}

\begin{tabular}{cccccc}
\hline No & $\mathrm{T} /{ }^{\circ} \mathrm{C}$ & {$[\mathrm{Cu}(\mathrm{I}) \mathrm{Br} / \mathrm{PMDETA}]_{0} / \mathrm{mM}$} & {$[\mathrm{ClPN}]_{0} / \mathrm{mM}$} & {$[\mathrm{TEMPO}]_{0} / \mathrm{mM}$} & $k_{\mathrm{obs}} / \mathrm{s}^{-1}$ \\
\hline $\mathrm{FS} 102$ & 20 & 2.26 & 23.1 & 23.0 & $2.03 \times 10^{-3}$ \\
$\mathrm{FS} 264$ & 20 & 4.94 & 127.3 & 50.3 & $1.48 \times 10^{-2}$ \\
$\mathrm{FS} 265$ & 20 & 4.71 & 202.2 & 48.0 & $2.33 \times 10^{-2}$ \\
\hline
\end{tabular}




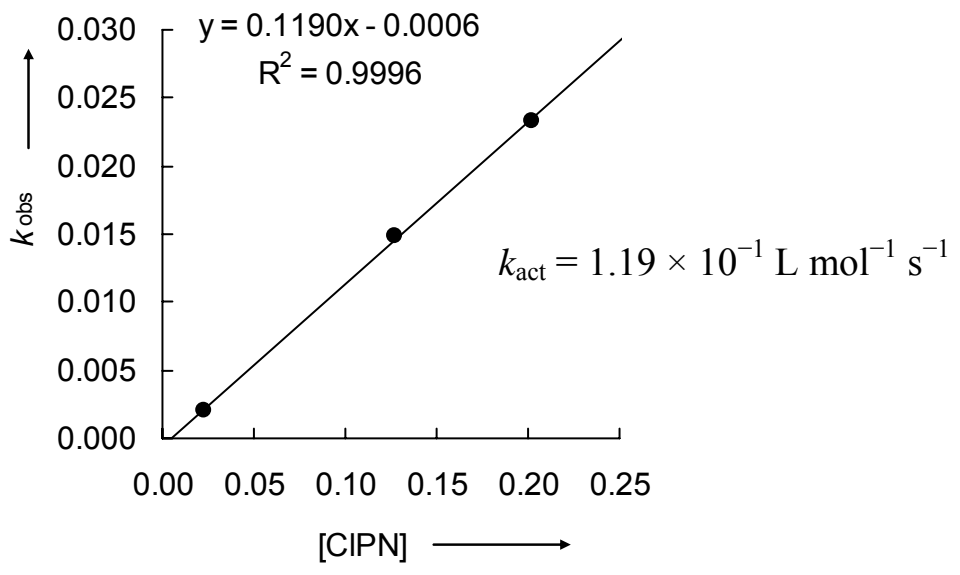

\begin{tabular}{ccccccc}
\hline No & $\mathrm{T} /{ }^{\circ} \mathrm{C}$ & {$[\mathrm{Cu}(\mathrm{I}) \mathrm{Br} / \mathrm{PMDETA}]_{0} / \mathrm{mM}$} & {$[\mathrm{ClPN}]_{0} / \mathrm{mM}$} & {$[\mathrm{TEMPO}]_{0} / \mathrm{mM}$} & $k_{\mathrm{obs}} / \mathrm{s}^{-1}$ & $k_{\mathrm{act}} / \mathrm{M}^{-1} \mathrm{~s}^{-1}$ \\
\hline $\mathrm{FS} 286$ & -20 & 4.89 & 257.0 & 49.6 & $2.40 \times 10^{-3}$ & $9.43 \times 10^{-3}$ \\
$\mathrm{FS} 285$ & 0 & 4.77 & 219.2 & 48.1 & $8.42 \times 10^{-3}$ & $3.84 \times 10^{-3}$ \\
$\mathrm{FS} 277$ & 40 & 3.31 & 35.0 & 34.6 & $8.49 \times 10^{-3}$ & $2.43 \times 10^{-1}$ \\
$\mathrm{FS} 278$ & 60 & 3.31 & 35.0 & 34.6 & $1.93 \times 10^{-2}$ & $5.52 \times 10^{-1}$ \\
\hline
\end{tabular}

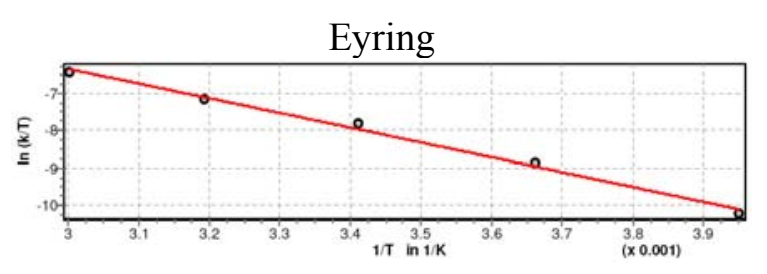

$$
\begin{gathered}
\Delta H^{\ddagger}=(32.9 \pm 1.4) \mathrm{kJ} \mathrm{mol}^{-1} \\
\Delta S^{\ddagger}=(-151 \pm 5) \mathrm{J} \mathrm{mol}^{-1} \mathrm{~K}^{-1}
\end{gathered}
$$

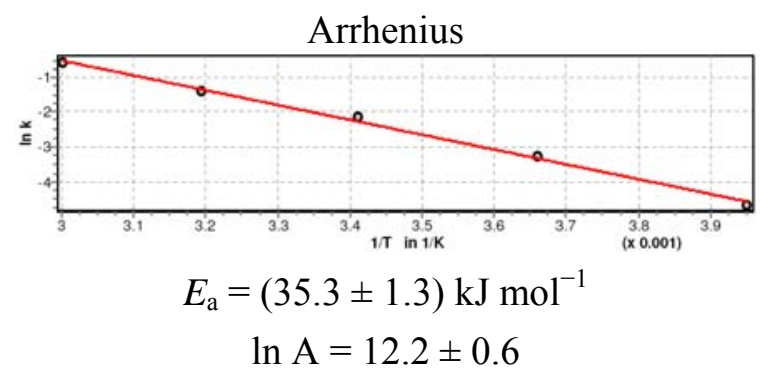

\section{PEBr}

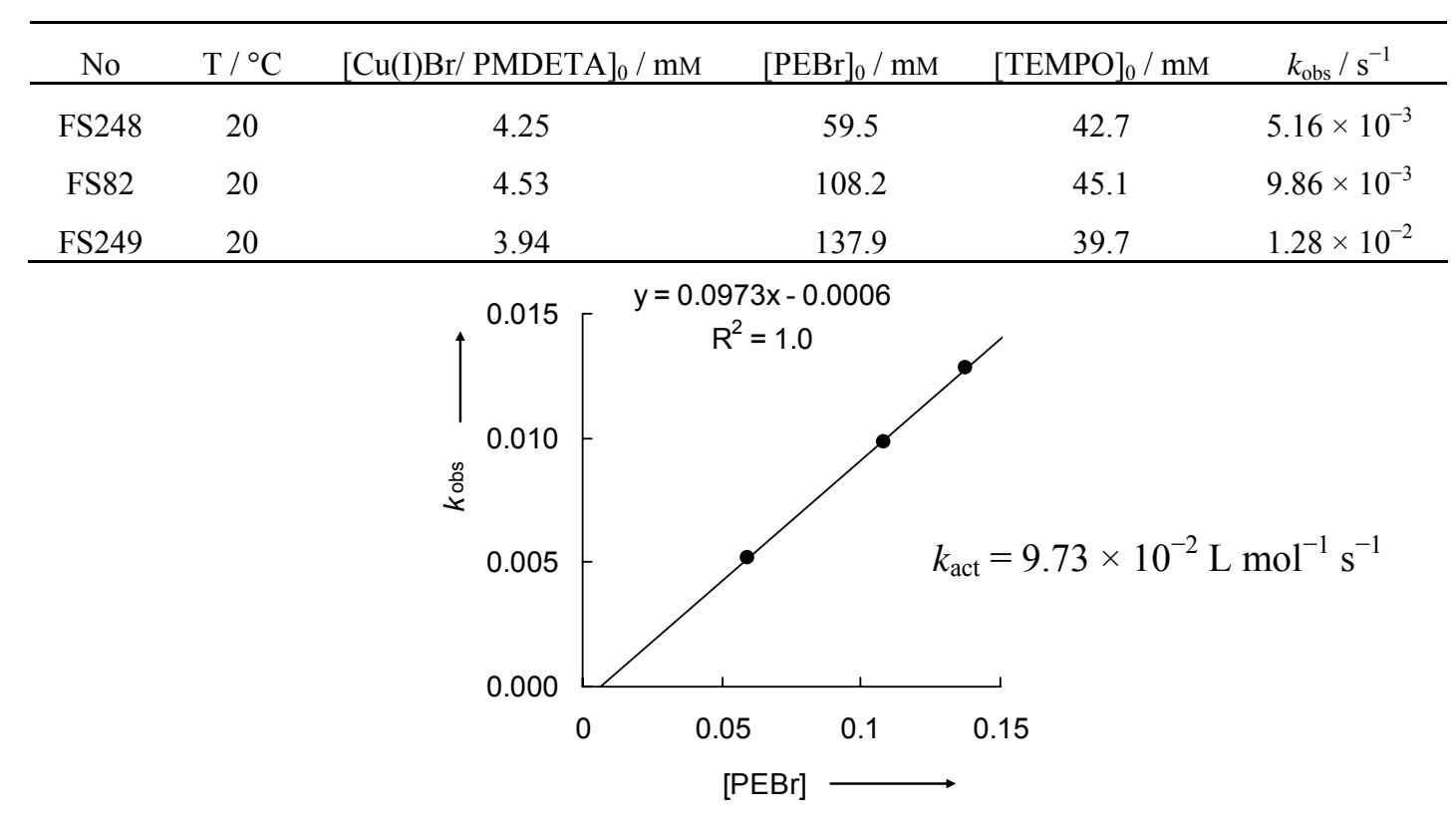




\begin{tabular}{ccccccc}
\hline No & $\mathrm{T} /{ }^{\circ} \mathrm{C}$ & {$[\mathrm{Cu}(\mathrm{I}) \mathrm{Br} / \mathrm{PMDETA}]_{0} / \mathrm{mM}$} & {$[\mathrm{PEBr}]_{0} / \mathrm{mM}$} & {$[\mathrm{TEMPO}]_{0} / \mathrm{mM}$} & $k_{\mathrm{obs}} / \mathrm{s}^{-1}$ & $k_{\mathrm{act}} / \mathrm{M}^{-1} \mathrm{~s}^{-1}$ \\
\hline FS78 & -20 & 4.05 & 82.9 & 40.8 & $8.18 \times 10^{-4}$ & $9.87 \times 10^{-3}$ \\
FS80 & 0 & 4.53 & 108 & 45.1 & $3.12 \times 10^{-3}$ & $2.88 \times 10^{-2}$ \\
FS84 & 40 & 4.05 & 61.9 & 40.6 & $1.53 \times 10^{-2}$ & $2.47 \times 10^{-1}$ \\
FS86 & 60 & 4.16 & 45.7 & 41.7 & $2.78 \times 10^{-2}$ & $6.08 \times 10^{-1}$ \\
\hline
\end{tabular}

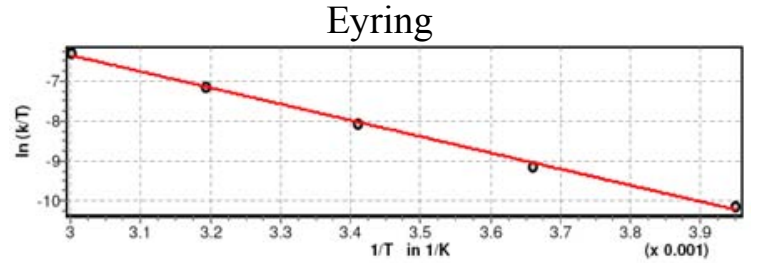

$$
\Delta H^{\ddagger}=(34.0 \pm 1.0) \mathrm{kJ} \mathrm{mol}^{-1}
$$$$
\Delta S^{\ddagger}=(-148 \pm 3) \mathrm{J} \mathrm{mol}^{-1} \mathrm{~K}^{-1}
$$

Arrhenius

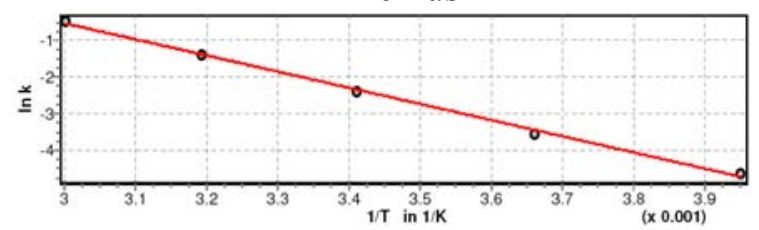

$E_{\mathrm{a}}=(36.4 \pm 1.0) \mathrm{kJ} \mathrm{mol}^{-1}$

$\ln \mathrm{A}=12.6 \pm 0.4$

\section{CIAN}

\begin{tabular}{cccccc}
\hline No & $\mathrm{T} /{ }^{\circ} \mathrm{C}$ & {$[\mathrm{Cu}(\mathrm{I}) \mathrm{Br} / \mathrm{PMDETA}]_{0} / \mathrm{mM}$} & {$[\mathrm{ClAN}]_{0} / \mathrm{mM}$} & {$[\mathrm{TEMPO}]_{0} / \mathrm{mM}$} & $k_{\mathrm{obs}} / \mathrm{s}^{-1}$ \\
\hline TM17 & 20 & 4.37 & 81.4 & 44.9 & $4.62 \times 10^{-3}$ \\
$\mathrm{TM} 18$ & 20 & 4.24 & 126.3 & 43.6 & $7.31 \times 10^{-3}$ \\
$\mathrm{FS} 89$ & 20 & 4.33 & 170.7 & 45.0 & $9.88 \times 10^{-3}$ \\
\hline
\end{tabular}

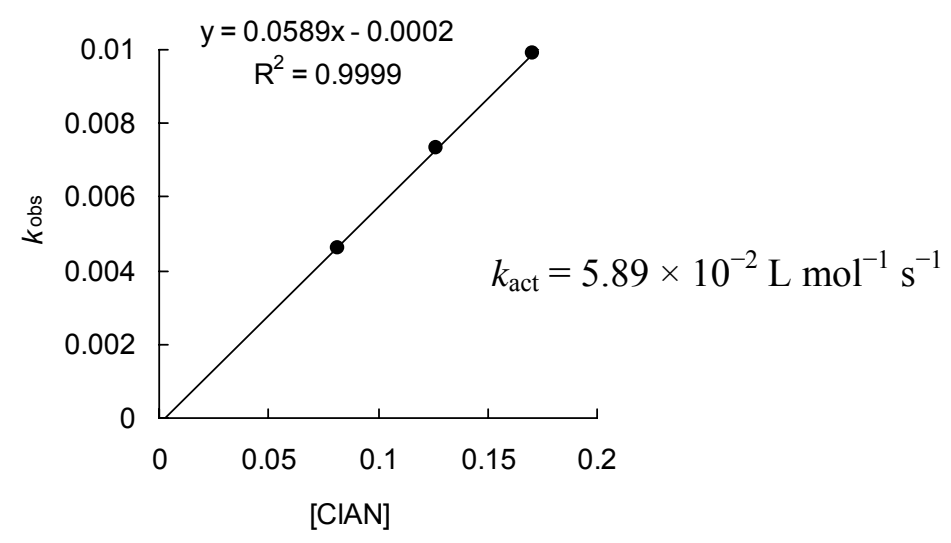

\begin{tabular}{ccccccc}
\hline No & $\mathrm{T} /{ }^{\circ} \mathrm{C}$ & {$[\mathrm{Cu}(\mathrm{I}) \mathrm{Br} / \mathrm{PMDETA}]_{0} / \mathrm{mM}$} & {$[\mathrm{ClAN}]_{0} / \mathrm{mM}$} & {$[\mathrm{TEMPO}]_{0} / \mathrm{mM}$} & $k_{\mathrm{obs}} / \mathrm{s}^{-1}$ & $k_{\mathrm{act}} / \mathrm{M}^{-1} \mathrm{~s}^{-1}$ \\
\hline FS87 & -20 & 4.30 & 314 & 43.0 & $1.60 \times 10^{-3}$ & $5.10 \times 10^{-3}$ \\
$\mathrm{FS} 88$ & 0 & 4.30 & 314 & 43.0 & $5.36 \times 10^{-3}$ & $1.71 \times 10^{-2}$ \\
FS90 & 40 & 3.30 & 65.6 & 33.0 & $8.91 \times 10^{-3}$ & $1.36 \times 10^{-1}$ \\
FS91 & 60 & 3.37 & 39.1 & 33.7 & $1.14 \times 10^{-2}$ & $2.92 \times 10^{-1}$ \\
\hline
\end{tabular}



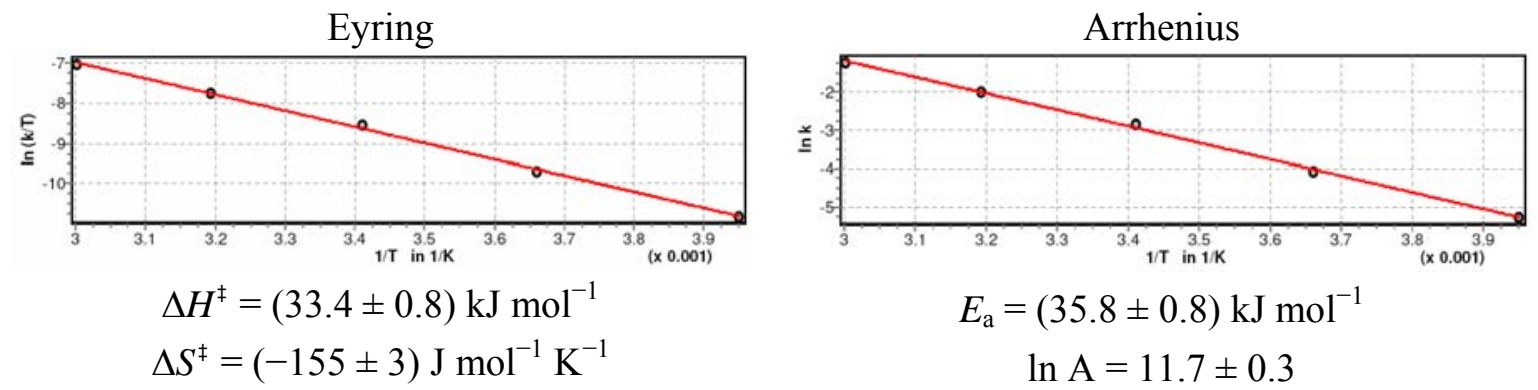

\section{AllBr}

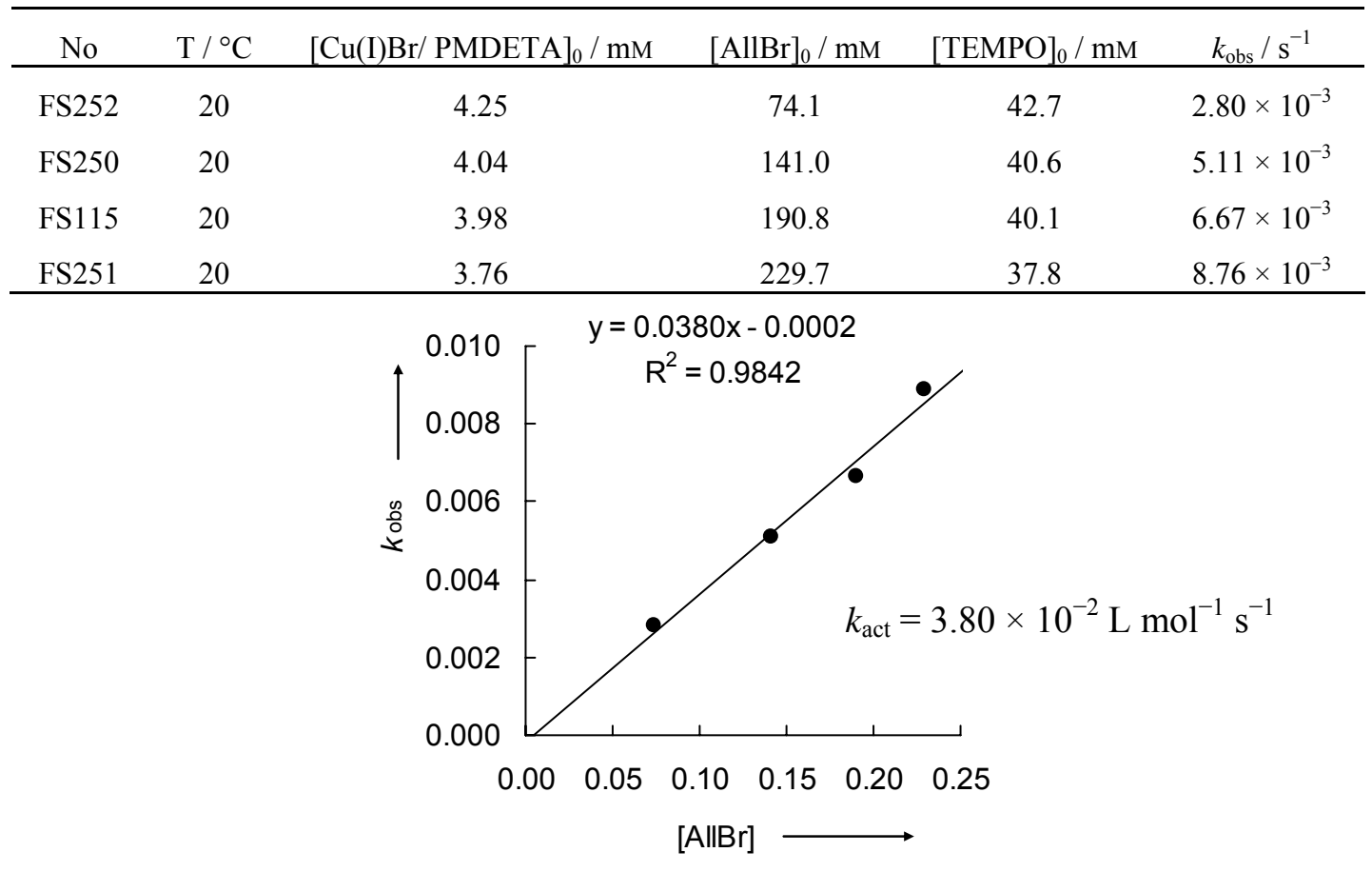

\begin{tabular}{ccccccc}
\hline No & $\mathrm{T} /{ }^{\circ} \mathrm{C}$ & {$[\mathrm{Cu}(\mathrm{I}) \mathrm{Br} / \mathrm{PMDETA}]_{0} / \mathrm{mM}$} & {$[\mathrm{AllBr}]_{0} / \mathrm{mM}$} & {$[\mathrm{TEMPO}]_{0} / \mathrm{mM}$} & $k_{\mathrm{obs}} / \mathrm{s}^{-1}$ & $k_{\mathrm{act}} / \mathrm{M}^{-1} \mathrm{~s}^{-1}$ \\
\hline FS120 & -20 & 3.98 & 191 & 40.1 & $5.42 \times 10^{-4}$ & $2.84 \times 10^{-3}$ \\
$\mathrm{FS} 119$ & 0 & 3.98 & 191 & 40.1 & $1.74 \times 10^{-3}$ & $9.12 \times 10^{-3}$ \\
$\mathrm{FS} 117$ & 40 & 4.20 & 126 & 42.4 & $1.32 \times 10^{-2}$ & $1.05 \times 10^{-1}$ \\
$\mathrm{FS} 118$ & 60 & 4.20 & 126 & 42.4 & $3.74 \times 10^{-2}$ & $2.97 \times 10^{-1}$ \\
\hline
\end{tabular}

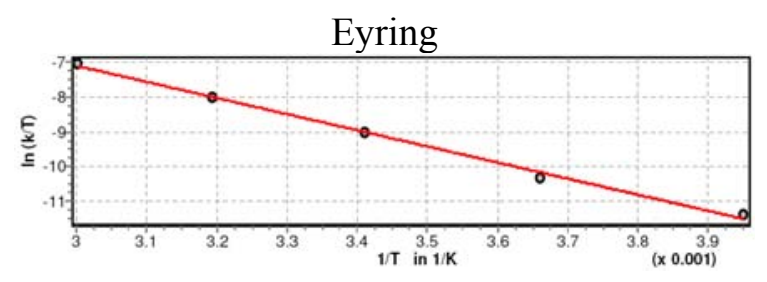

$$
\Delta H^{\ddagger}=(38.7 \pm 1.2) \mathrm{kJ} \mathrm{mol}^{-1}
$$$$
\Delta S^{\ddagger}=(-140 \pm 4) \mathrm{J} \mathrm{mol}^{-1} \mathrm{~K}^{-1}
$$

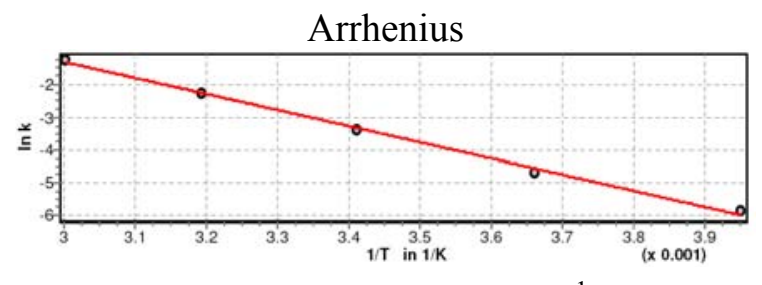

$E_{\mathrm{a}}=(41.1 \pm 1.3) \mathrm{kJ} \mathrm{mol}^{-1}$

$\ln \mathrm{A}=13.6 \pm 0.5$ 


\section{MBrP}

\begin{tabular}{cccccc}
\hline No & $\mathrm{T} /{ }^{\circ} \mathrm{C}$ & {$[\mathrm{Cu}(\mathrm{I}) \mathrm{Br} / \mathrm{PMDETA}]_{0} / \mathrm{mM}$} & {$[\mathrm{MBrP}]_{0} / \mathrm{mM}$} & {$[\mathrm{TEMPO}]_{0} / \mathrm{mM}$} & $k_{\mathrm{obs}} / \mathrm{s}^{-1}$ \\
\hline $\mathrm{FS} 111$ & 20 & 4.00 & 40.1 & 42.3 & $5.51 \times 10^{-3}$ \\
$\mathrm{FS} 110$ & 20 & 3.85 & 77.2 & 40.6 & $1.01 \times 10^{-2}$ \\
$\mathrm{FS} 109$ & 20 & 3.71 & 111 & 39.2 & $1.56 \times 10^{-2}$ \\
$\mathrm{FS} 108$ & 20 & 3.58 & 143 & 37.8 & $1.98 \times 10^{-2}$ \\
$\mathrm{FS} 112$ & 20 & 3.45 & 173 & 36.5 & $2.29 \times 10^{-2}$ \\
\hline
\end{tabular}

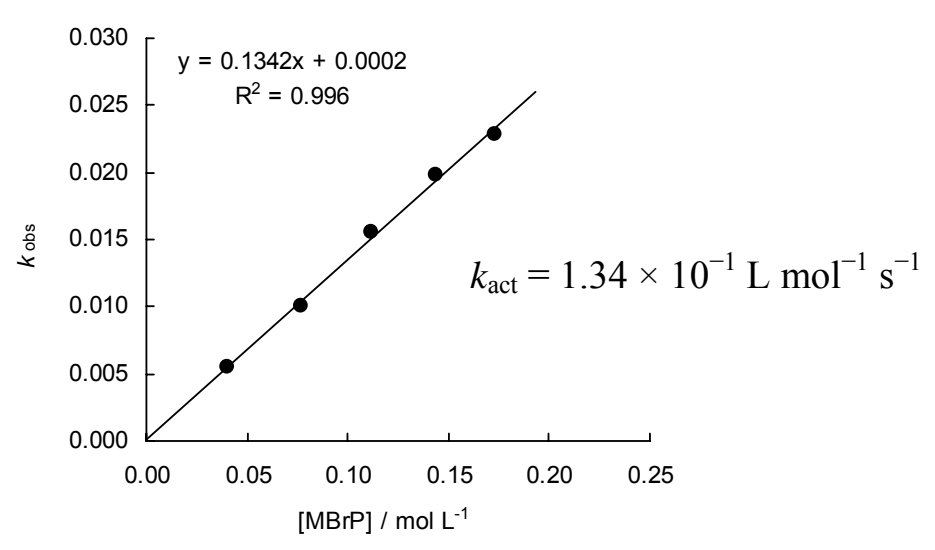

\begin{tabular}{ccccccc}
\hline No & $\mathrm{T} /{ }^{\circ} \mathrm{C}$ & {$[\mathrm{Cu}(\mathrm{I}) \mathrm{Br} / \mathrm{PMDETA}]_{0} / \mathrm{mM}$} & {$[\mathrm{MBrP}]_{0} / \mathrm{mM}$} & {$[\mathrm{TEMPO}]_{0} / \mathrm{mM}$} & $k_{\mathrm{obs}} / \mathrm{s}^{-1}$ & $k_{\mathrm{act}} / \mathrm{M}^{-1} \mathrm{~s}^{-1}$ \\
\hline FS77 & -20 & 4.05 & 62.1 & 40.8 & $9.95 \times 10^{-4}$ & $1.60 \times 10^{-2}$ \\
FS79 & 0 & 4.45 & 66.0 & 44.3 & $3.75 \times 10^{-3}$ & $5.68 \times 10^{-2}$ \\
FS83 & 40 & 3.30 & 35.2 & 33.1 & $1.20 \times 10^{-2}$ & $3.41 \times 10^{-1}$ \\
FS85 & 60 & 3.98 & 40.7 & 39.9 & $2.96 \times 10^{-2}$ & $7.27 \times 10^{-1}$ \\
\hline
\end{tabular}
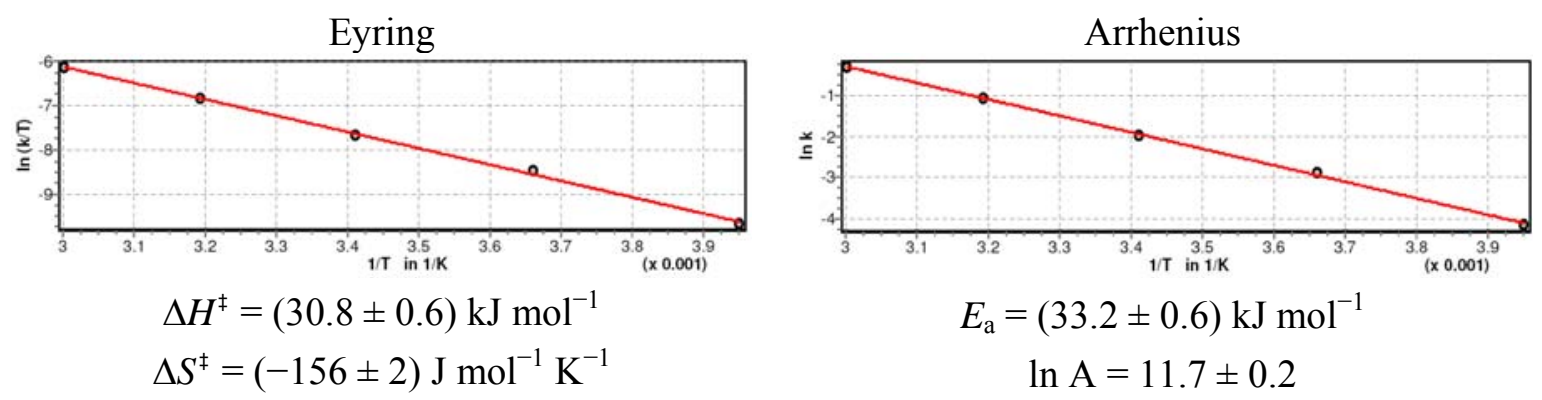


\section{Determination of $\Delta \boldsymbol{S}^{\ddagger}$}

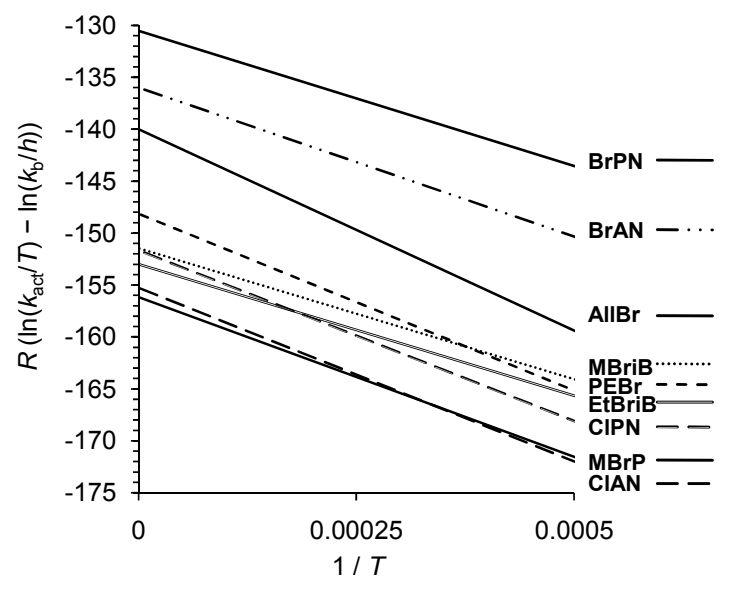

Determination of the entropy of activation $\left(\Delta S^{\ddagger}\right)$ for studied reactions by extrapolation to infinite temperatures $(1 / T \rightarrow 0)$. 